\title{
Visualization of the Impact of Oxygen Adsorption on Spin Reorientation Transition of a Ferromagnetic Ultrathin Film by X-ray Photoemission Electron Microscopy.
}

\author{
Tzu-Hung Chuang ${ }^{1, *}$, Chih-Heng Huang ${ }^{2}$, Chun-I Lu' ${ }^{1}$, and Der-Hsin Wei ${ }^{1,2}$ \\ 1. National Synchrotron Radiation Research Center, Hsinchu, Taiwan. \\ 2. Program for Synchrotron Radiation and Neutron Beam Applications, National Sun Yat-sen University, \\ Kaohsiung, Taiwan. \\ * Corresponding author, chuang.th@ nsrrc.org.tw
}

The investigation of magnetic properties of thin films and surfaces has led to important technological applications, such as magnetic recording techniques, etc. Of particular importance for both fundamental research and possible applications in magnetic recording devices is the influence of the temperature, film thickness or the film composition on the direction of the magnetization. Spin reorientation transitions (SRT), where the magnetization direction is changed as a function of the above mentioned parameters, have been intensively studied. The orientation of the magnetization is determined by the magnetic anisotropy energy, which includes spin-orbit (magnetocrystalline and magnetostrictive anisotropy) and dipolar (shape anisotropy) induced interactions.

Ultrathin $\mathrm{Fe} / \mathrm{Ni}$ bilayer grown on $\mathrm{Cu}(100)$ single crystal represents a prototype of a ferromagnetic double layer system, where the individual layers exhibit an opposite SRT [1]. The magnetization of ultrathin $\mathrm{Fe} / \mathrm{Cu}(100)$ proceeds from out-of-plane to in-plane at a thickness of about 4-5 monolayer (ML) at $300 \mathrm{~K}$, while the magnetization of ultrathin $\mathrm{Ni} / \mathrm{Cu}(100)$ is switched from in-plane to out-of-plane at 7-8 ML, which is a reversed SRT in comparison of $\mathrm{Fe} / \mathrm{Cu}(100)$ [2-3]. The reason is due to a negative surface anisotropy of $\mathrm{Ni}$, which favours a spin orientation in the plane and has the largest contribution at low thickness. Macroscopic magnetic properties of $\mathrm{Fe} / \mathrm{Ni} / \mathrm{Cu}(100)$ have been investigated by magneto-optic Kerr effect (MOKE) and shown a SRT from out-of-plane to in-plane [1]. Microscopic imaging of magnetic domain structures in epitaxial $\mathrm{Fe} / \mathrm{Ni} / \mathrm{Cu}(100)$ has been investigated by $\mathrm{X}$-ray photoemission electron microscopy (XPEEM) [4] and spin-polarised low-energy electron microscopy [5], and has found a stripedomain feature across SRT region. It has also been studied by MOKE that the oxygen adsorption on a ferromagnetic ultrathin film can modulate the surface anisotropy and has impact on the SRT thickness [6]. In this report, we provide a microscopic insight on how the oxygen adsorption modulates the SRT of a $\mathrm{Fe} / \mathrm{Ni}$ bilayer on $\mathrm{Cu}(100)$ and visualize the evolution of the featured stripe-domains across SRT at different thicknesses of oxygen coverage by XPEEM.

The experiments were performed at the XPEEM end-station (BL05B2) at the Taiwan Light Source. All images were recorded with a circular polarized X-ray beam with an incident angle of $65^{\circ}$ to the surface normal at room temperature. A detail description of the end-station can be found elsewhere [7]. The Fe/Ni bilayers were grown epitaxially with a base pressure around $5 \times 10^{-10}$ torr. The Fe and Ni ultrathin films were deposited at a rate of $0.67 \mathrm{ML}$ per minute (calibrated independently with the oscillation of mediumenergy electron diffraction intensity of the specular beam during deposition on a clean $\mathrm{Cu}(100)$ ). The $\mathrm{Ni}$ layer was deposited with a single thickness (5 ML) on $\mathrm{Cu}(100)$, then an Fe wedge from 1 to $3 \mathrm{ML}$ with a slope of $2 \mathrm{ML} / \mathrm{mm}$ was then deposited on top (the sample structure is shown in the inset of Fig. 1).

In Fig. 1, a series of magnetic domain images on a Fe wedge thin film (1-3 ML) grown on $5 \mathrm{ML} \mathrm{Ni/Cu(100)}$ across SRT and its evolution by oxygen adsorption ( 0.25 and 0.5 Langmuir (L)) have been recorded by 
taking the ratio of $\mathrm{Fe}_{3}$ and $\mathrm{L}_{2}$ edges, utilizing X-ray magnetic circular dichroism (XMCD) contrast (outof-plane magnetization with thinner film at right side of Fig. 1 to in-plane magnetization with thicker film at left side). There are only two intensity-contrasts at the right side of the figure, which is in line with the result of out-of-plane magnetization of thinner films. The broken and striped domains were found at about 2.61 ML of Fe, where the thickness is determined by the relative location on the wedge. With $0.25 \mathrm{~L}$ of oxygen adsorption, the SRT shifts to about $2.55 \mathrm{ML}$, and with $0.50 \mathrm{~L}$ adsorption, the SRT further moves to about $2.49 \mathrm{ML}$. Only $0.50 \mathrm{~L}$ of oxygen coverage is able to modify the surface anisotropy and cause around 5\% changes at SRT thickness (2.61 to 2.49 ML), suggesting that the SRT is extremely sensitive to the oxygen adsorption, which has been found to induce structural changes and to decrease the magnetocrystalline anisotropy [6]. Therefore, the shape anisotropy, which favors in-plane magnetization in the case of thin film, dominates the magnetocrystalline anisotropy with oxygen adsorption.

In summary, a series of magnetic domain images at $\mathrm{SRT}$ of $\mathrm{Fe} / \mathrm{Ni} / \mathrm{Cu}(100)$ and its evolution by oxygen adsorption were clearly recorded by XMCD-PEEM. The change of SRT thickness towards thinner Fe films due to the decrease of magnetocrystalline anisotropy by oxygen was visualized by the shifting of stripe-domain region, while the domain features at other part remain unchanged. The result provides a microscopic picture of the competition between magnetocrystalline anisotropy and shape anisotropy [8].

References:

[1] X. Liu and M. Wuttig, Phys. Rev. B 64, 104408 (2001).

[2] D. Peterka, et al, Phys. Rev. B 66, 104411 (2002).

[3] W. L. O’Brien and B. P. Tonner, Phys. Rev. B 49, 15370 (1994).

[4] Y. Z. Wu, et al, Phys. Rev. Lett. 93, 117205 (2004).

[5] R. Ramchal, et al, Phys. Rev. B 69, 214401 (2004).

[6] J. Chen, M. Drakaki, and J. L. Erskine, Phys. Rev. B 45, 3636 (1992).

[7] D.-H. Wei, Y.-L. Chen, and Y.-J. Hsu, J. Electron. Spectrosc. Relat. Phenom. 185, 429 (2012).

[8] The authors acknowledge funding from the Ministry of Science and Technology in Taiwan (MOST 105-2112-M-213 -012 -MY2).

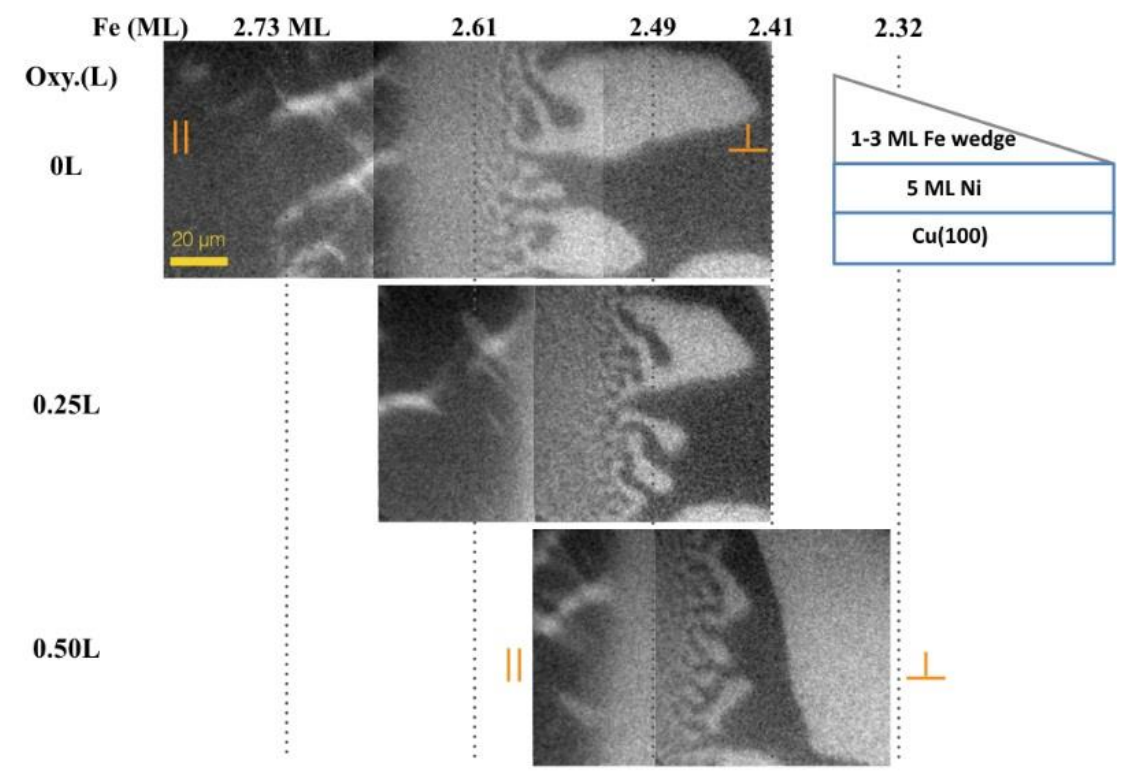

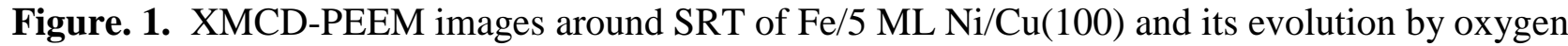
passivation ( 0 to $0.5 \mathrm{~L}$ ). The scale bar of the images is $20 \mu \mathrm{m}$. The structure of the sample is shown in the inset. All images were obtained by taking the ratio of $\mathrm{Fe} \mathrm{L}_{3}$ and $\mathrm{L}_{2}$ edges at $300 \mathrm{~K}$. 